\title{
THERMAL AND HALL-CURRENT INSTABILITIES IN A CURRENT SHEET AND THE TRIGGERING OF TWO RIBBON FLARES
}

\section{Li Sang Jae}

Pyongyang Astronomical Observatory, Academy of Sciences, Democratic People's Republic of Korea

\begin{abstract}
Thermal stability of a current sheet is investigated when the magnetic field is not perfectly anti-parallel at the sheet. If the effect of Hall electric current is taken into account, a new instability (the Hall instability) appears. The result is applied to the activation of dark filaments often seen prior to solar flares. The growth time of the instability is evaluated and found to be consistent with the observed time scale of the filament activation.
\end{abstract}

\section{INTRODUCTION}

The ratio between the Hall current and the Ohmic current is represented as the product of the electron gyro frequency and the electron-ion collision time as $\Omega_{e} \tau_{e}$. This quantity, the Hall index, takes a very large value in space plasmas (TABLE 1). One may expect that the Hall current may play an important role in the solar atmosphere.

Table 1. Hall index $\left(\Omega_{e} \tau_{e}\right)$ in space plasmas

$$
B \text { (Gauss) } \quad N_{\mathrm{e}}\left(\mathrm{cm}^{-3}\right) \quad T_{\mathrm{e}}(\mathrm{K}) \quad \Omega_{\mathrm{e}} \tau_{\mathrm{e}}
$$

$\begin{array}{lllll}\begin{array}{l}\text { Sun: } \\ \text { lower chromosphere }\end{array} & 10^{2-3} & 10^{12-13} & 10^{4} & 10^{1-2} \\ \begin{array}{l}\text { upper chromosphere } \\ \text { lower corona }\end{array} & 10^{2} & 10^{10-11} & 10^{5} & 10^{5-6} \\ \begin{array}{l}\text { upper corona } \\ \text { near earth }\end{array} & 10 & 10^{9-10} & 10^{6} & 10^{6-7} \\ \text { magnetosphere } & 10^{-5} & 10^{8} & 10^{6} & 10^{7} \\ & 1 & 10^{4} & 10^{6} & 10^{8} \\ & & & 10^{4} & 10^{8}\end{array}$

Note: $B$ is the magnetic field, $N_{\mathrm{e}}$ is the electron density, $T_{\mathrm{e}}$ is the electron temperature, $\Omega_{\mathrm{e}}$ is the electron gyro frequency, and $\tau_{\mathrm{e}}$ is the electron-ion collision time. 
In the case of the Rayleigh-Taylor instability, the Hall current and finite electrical conductivity are the factors to drive the instability (Ogbonna and Bhatia 1984). On the other hand it is well known that finite conductivity causes the tearing instability in a current sheet (Furth et al. 1963), and this instability may play an important role in solar flares (Spicer 1981). One may expect a similar contribution from the Hall current to the instability in the current sheet.

It is also important to notice that the current sheet is not neutral in general. In addition to the anti-parallel magnetic field (APMF), the current sheet will also have a longitudinal component of the magnetic field ( $\mathrm{LMF}$, the component which does not change sign across the current sheet)(Somov 1985). However, the effect of the LMF has not been fully studied yet.

In this paper, we will study the instability of the current sheet by taking in to account the effects of the Hall current and the LMF, and discuss a possible role of the instability on the triggering of solar flares.

\section{THERMAL AND HALL INSTABILITIES OF A CURRENT SHEET}

If the effects of the LMF and the Hall current are taken into account, the thermal instability in current sheets studied by Somov and Syrovatskii (1982) is modified in two ways, as will be shown below. In the present study, the electrical conductivity is assumed to be infinitely high.

First we ignore the Hall current. The maximum growth rate of the thermal instability in this case is

$$
\begin{aligned}
\Gamma_{\max }=\frac{1}{C_{\mathrm{s} 2}^{2}-V_{\mathrm{A} 2}^{2}} & \left\{\tau_{\mathrm{r}}^{-1}\left[C_{\mathrm{s} 2}^{2}(2-\alpha(T))+\gamma V_{\mathrm{A} 2}^{2} \alpha(T)\right]\right. \\
& \left.-\tau_{\kappa}^{-1}\left(C_{\mathrm{s} 2}^{2}-\gamma V_{\mathrm{A} 2}^{2}\right)\right\}, \quad C_{\mathrm{s} 1}>V_{\mathrm{A} 1}
\end{aligned}
$$

where

$$
\begin{gathered}
\alpha(T)=d \ln L(T) / d \ln T, \\
\tau_{\mathrm{r}}=\frac{\gamma}{\gamma-1} \frac{2 k T}{N_{0} L(T)}, \\
\tau_{\kappa}=\frac{\gamma}{\gamma-1} \frac{2 k N_{0}}{\kappa_{0} T^{5 / 2}} \frac{B_{0}^{2}}{\left(\boldsymbol{K} \cdot B_{0}\right)^{2}}, \\
C_{\mathrm{s}}^{2}=\gamma \frac{2 k T}{\mu} . \\
V_{\mathrm{A}}^{2}=\frac{B_{0}^{2}}{4 \pi \mu N_{0}} .
\end{gathered}
$$

Here $\boldsymbol{B}_{\mathbf{0}}, N_{0}, T$ are the magnetic field, the number density, and the temperature of the unperturbed state, respectively, and $K$ and $\Gamma$ are the wavenumber and the growth rate of the perturbation. $L(T)$ is the radiative loss function, $\kappa_{0} T^{5 / 2}$ is the thermal conductivity, and $\gamma$ is the ratio of specific heats. Indices 1 and 2 are used for the quantities outside and inside of the current sheet, respectively.

It is found that the instability is completely suppressed for $C_{\mathrm{s} 1}<V_{\mathrm{A} 1}$, i.e. for large enough LMF. That is, the thermal instability obtained by Somov 
and Syrovatskii (1982) has a threshold value for the LMF. If the LMF is above the threshold, the instability is suppressed. As long as the LMF is below the threshold, the growth rate is larger for larger LMF.

Secondly, in the case of incompressible perturbations, the system is unstable due to the combined effect of the Hall current and the LMF. The maximum growth rate in this case is

$$
\begin{aligned}
& \Gamma_{\max } \simeq K V_{\mathrm{A} 2} \quad \text { for } \quad a>a_{0}, \\
& \frac{2}{\pi} a K \Omega_{2}<\Gamma_{\max }<K V_{\mathrm{A} 2} \quad \text { for } a<a_{0} \text {, }
\end{aligned}
$$

where

$$
\Omega=\frac{e B_{0}}{\mu c}, \quad a_{0}=\frac{\pi}{2 \sqrt{2}} \frac{V_{\mathrm{A} 2}}{\Omega_{2}},
$$

and $a$ is the half thickness of the sheet. For typical solar flares we adopt $N_{0}=$ $10^{11} \mathrm{~cm}^{-3}, a_{0}=300 \mathrm{~cm}$, and $B_{0}=10 \mathrm{G}$. If we assume that the maximum wavelength of perturbation along the current sheet is $l=2 \pi / K_{\min } \simeq 10^{10} \mathrm{~cm}$, the growth time of the Hall instability $\left(\tau=\Gamma_{\max }^{-1}\right)$ turns out to be $\tau=300 \mathrm{~s}$ for $a>300 \mathrm{~cm}$ and $10^{3} \mathrm{~s}>\tau>300 \mathrm{~s}$ for $a \simeq 30 \mathrm{~cm}$. This time scale agrees with the duration of the pre-flare activity of dark filaments.

\section{EXPLOSION OF DARK FILAMENTS AND THE TRIGGERING OF} TWO-RIBBON FLARES

As it is widely known, two-ribbon flares arise generally on the magnetic arcade in which a dark filament is located on the photospheric magnetic neutral line. It is common that the activity in the dark filament continues for about $10 \mathrm{~min}$, and then the dark filament explodes and a two-ribbon flare takes place.

One possible explanation for these phenomena is given in terms of the kink instability (Hood and Priest 1980). However, this explanation does not give adequate account on the pre-flare activity of dark filaments. Another possibility is the thermal instability. It was suggested (Somov and Syrovatskii 1982; Somov 1987) that two-ribbon flares are triggered when the axial current in the filament becomes unstable against the thermal instability. However, the expected growth time of the thermal instability is too short $\left(0.1-10 \mathrm{~s}\right.$ for $N_{02}=10^{11} \mathrm{~cm}^{-3}$ and $\left.T_{02}=10^{5-6} \mathrm{~K}\right)$ to acount for the observed slow activation of the dark filaments.

A more realistic estimate for the time scale of pre-flare filament activation can be obtained by considering the Hall instability and the stabilizing effect of the LMF. Figure 1 depicts the current sheet in the plane that includes the electric current and the LMF. (The APMF is perpendicular to the plane of the paper.) If $B_{0 \mathrm{z}} \geq 7 \mathrm{G}\left(N_{0}=10^{10} \mathrm{~cm}^{-3}, T_{0}=10^{6} \mathrm{~K}\right)$, the condition $C_{\mathrm{s} 1}<V_{\mathrm{A} 1}$ is satisfied and consequently the currnet sheet is stable against the thermal instability (figure 1a). If there is shear in the magnetic field so that $B_{\mathrm{z} 1}>B_{\mathrm{z} 2}$ holds, the Hall instability might be excited and the current sheet will take the form as depicted in figure $1 \mathrm{~b}$. From the adiabatic relation and the conservations of mass and magnetic flux, it can be shown that the condition $C_{\mathbf{s 1}}>V_{\mathrm{A} 1}$ is satisfied in part II of the sheet. Therefore the the current sheet is now unstable to the thermal instability, and will explode as it evolves into the nonlinear stage 
(Somov 1987). The duration of the pre-flare activity of dark filaments is also explained in this scenario.

\section{CONCLUDING REMARKS}

We have studied the stability of a current sheet by considering both the LMF and the Hall current. If the mechanism mentioned above is realized in the solar atmosphere, we expect two kinds of solar flares. If the LMF is weak $\left(B_{z}<10 \mathrm{G}\right)$, the flare will take place without the activity of the dark filament. If the LMF is larger, the flare is associated with the activity of the dark filament for about 10 minutes, due to the Hall instability. The number of flares without filament activation will only be $3 \%$ of the total.

The present analysis has adopted a very simplified model for the current sheet. The next step is to use, for example, the Harris profile $\left(B_{0 x}(y) \sim\right.$ $\tanh (y / a))$. It is also important to study the relation between the instabilities studied here and the tearing mode instability. A full account of the stability analysis outlined in section 2 will be given elsewhere (Li 1992).

\section{REFERENCES}

Furth, H.P., Kileen, J., and Rosenbluth, Monthly Notices Roy. Astron. Soc., 1963, Phys. Fluids, 6, 459.

Hood, A.W., and Priest, E.R. 1980, Solar Phys., 66, 113.

Li, S.J. 1992, to be submitted to Solar Phys.

Ogbonna, N., and Bhatia, P.K. 1984, Astrophys. Space Sci., 103, 9.

Spicer, D.S. 1981, Solar Phys., 70, 147.

Somov, B.V. 1987, Itogi Nauki Tech. Astron. 34, 50.

Somov, B.V., and Syrovatskii, S.I. 1982, Solar Phys., 45, 237.

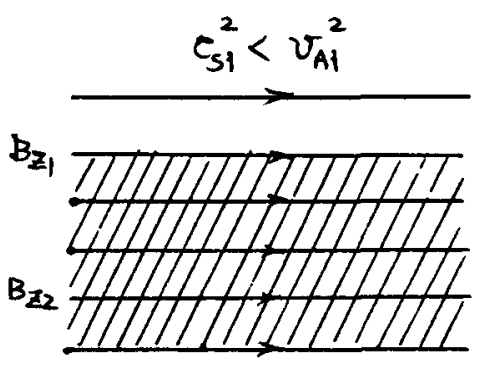

(a) Equilibrium

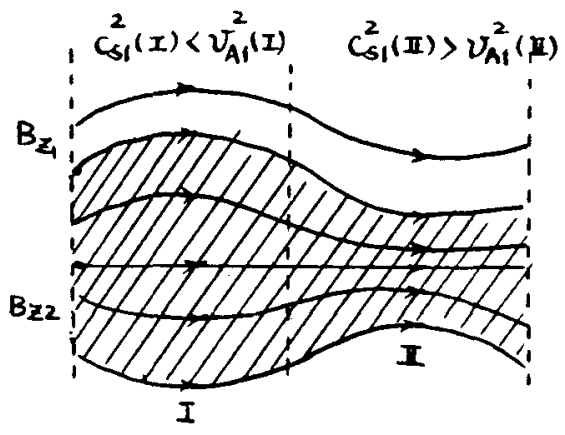

(b) A state with the Hall instability

Fig.1. The form of the current sheet and the magnetic field lines. 